Für alle Schweregrade der COPD

\title{
Lungensport ist ein Muss
}

— Die zunächst nur bei Belastung spürbare Atemnot verleitet COPD-Patienten bereits in einem frühen Stadium zu körperlicher Inaktivität. Folge ist eine zunehmende physische Dekonditionierung, die die Belastungsgrenze, ab der Atemnot auftritt, weiter senkt. Mit regelmäßigem körperlichen Training kann diese Negativspirale nicht nur verzögert, sondern sogar in erheblichem Umfang revidiert werden. Deswegen ist ein dem individuellen Leistungsniveau angepasstes regelmäßiges Kraft-, Ausdauer und Koordinationstraining für COPD-Patienten aller Schweregrade ein Muss, betonte Dr. Marc Spielmanns, Leverkusen.

\section{Bronchodilatatoren steigern den Trainingserfolg}

Mehrere Untersuchungen haben gezeigt, dass die durch körperliches Training erreichbaren Erfolge bezüglich Belastbarkeit, Dyspnoe und Lebensqualität schon im GOLD-Stadium II durch begleitenden

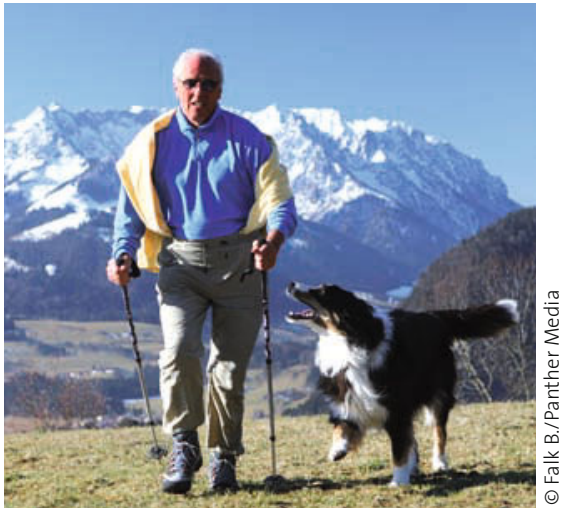

Mit Ausdauertraining der Dekonditionierung und stärkerer Atemnot entgegenwirken.

Einsatz von lang wirksamen Bronchodilatatoren wie Tiotropium (Spiriva ${ }^{\circledR}$ ) maßgeblich gesteigert werden können. Nationale und internationale Fachgesellschaften empfehlen deshalb schon $a b$ diesem Stadium zusätzlich zum Lungensport eine medikamentöse Basistherapie mit lang wirksamen Bronchodilatatoren.
Um Patienten möglichst früh im Verlauf einer COPD zu Lungensport und zur Aufgabe des Hauptrisikofaktors Rauchen zu motivieren, kann es hilfreich sein, sie einen kleinen Selbstversuch machen zu lassen, sagte Sporttherapeut Dr. Oliver Göhl, Bad Reichenhall. Dazu sollen sie mit zugehaltener Nase durch einen Strohhalm im Kaliber $5 \mathrm{~mm}$ (Obstruktionssimulation) konsequent in gehobener Atemmittellage (Emphysemsimulation) atmend erst eine halbe Minute sitzen und dann noch ein paar Treppenstufen steigen. Dabei bekommen sie eine eindrucksvolle Ahnung davon, wie sich eine fortgeschrittenere COPD anfühlt und vielleicht die Überzeugung: Es lohnt sich, etwas dafür zu tun, damit es nicht so weit kommt.

- Werner Stingl

Quelle: Fachpresse-Workshop „Mehr Aktivität für COPD-Patienten“, Bad Reichenhall, 18. Juni 2010 (Veranstalter: Boehringer Ingelheim und Pfizer)

\section{Experten kritisieren Verordnungspraxis bei Typ-2-Diabetes „First-Line-Therapie wie vor 50 Jahren“}

- Bei Typ-2-Diabetes empfehlen die Leitlinien nach wie vor einen $\mathrm{HbA}_{1 c}$-Wert unter $6,5 \%$, lediglich für ältere und multimorbide Patienten sind die Zielwerte etwas weniger streng. Die Behandlung sollte aber so erfolgen, dass die Patienten keine Hypoglykämien entwickeln und nicht zunehmen.,,Trotzdem erhalten 90\% der Patienten primär althergebrachte Medikamente wie die Sulfonylharnstoffe, mit denen die Ziele der modernen Diabetestherapie praktisch nicht zu realisieren sind“, monierte Dr. Ralph Bierwirth, Essen. „Wir praktizieren damit eine First-LineTherapie wie vor 50 Jahren."

\section{Erhöhte Mortalität durch Hypoglykämien}

Dabei wird nach seinen Worten in Kauf genommen, dass den Patienten eine er- höhte Mortalität durch Hypoglykämien droht. Die Gefahr durch Unterzuckerungen werde noch weitgehend unterschätzt. Eine erhöhte Glukagon- und auch eine erhöhte Adrenalinsekretion sind die Folge und im weiteren Verlauf eine verminderte Wahrnehmungsfähigkeit, Krämpfe, Koma und schließlich das Absterben von Hirnzellen.

Ferner ist laut Bierwirth Vorsicht geboten bei der Diabetestherapie mit insulinotropen Wirkstoffen, weil diese in aller Regel zu einer Gewichtssteigerung der meist per se schon übergewichtigen Patienten führen und damit das kardiovaskuläre Risiko weiter steigern.

„Wir müssen deshalb eine Therapie realisieren, die gewichtsneutral den Blutzucker kontrolliert, und das ohne die Gefahr von Hypoglykämien“, forderte Bier- wirth. Möglich ist dies durch inkretinbasierte Therapiestrategien wie die DPP-4Inhibitoren. Die meisten Erfahrungen liegen mit dem Wirkstoff Sitagliptin (z.B. Xelevia ${ }^{\circledR}$ ) vor, der unter den DPP-4-Inhibitoren auch über das breiteste Zulassungsspektrum verfügt. Sitagliptin kann als Monotherapie sowie in Kombination mit praktisch allen Antidiabetika und als Addon zu Insulin verabreicht werden. Besonders günstig ist laut Bierwirth die Kombinationstherapie mit Metformin (z.B. Velmetia $\left.{ }^{\circledR}\right)$, da die Substanzen komplementär wirken: Während Metformin die GLP-1Ausschüttung fördert, bewahrt Sitagliptin das blutzuckerregulierende Hormon davor, rasch wieder abgebaut zu werden.

\section{- Christine Vetter}

Quelle: Pressegespräch, Köln, 8. September 2010 (Veranstalter: Berlin-Chemie) 